\title{
Models and Mechanisms of Regenerative Biology Across Phylogeny: Introduction to a Virtual Symposium in The Biological Bulletin
}

\author{
JOEL SMITH ${ }^{1 * *}$ AND JAMES L. OLDS ${ }^{2} *$ \\ ${ }^{1}$ Eugene Bell Center for Regenerative Biology and Tissue Engineering, Marine Biological Laboratory, \\ Woods Hole, Massachusetts; and ${ }^{2}$ Department of Molecular Neuroscience, Krasnow Institute for \\ Advanced Study, George Mason University, Fairfax, Virginia
}

Within the animal context, biological regeneration can be defined as a spatio-temporal process whereby biological tissue is replaced or repaired, often without external intervention, and thence regains some or all of its original form and function. It shares some similarities to ontogeny both at the systems and molecular levels. In his 1901 work (Regeneration [Macmillan, NY]), Thomas Hunt Morgan distinguished between physiological regeneration and restorative regeneration. In physiological regeneration, the process occurs in the normal course of an animal's life cycle; in restorative regeneration an external injury is the trigger. The common factor, however, involves both the mobilization and movement of cells and, often at the same time, cell proliferation and cell death. A modern example of physiological regeneration is the phenomenon of neurogenesis in the hippocampus of the adult mammalian rodent (van Praag, H., et al., Nature 2002 415(6875):1030-4). An example of restorative regeneration can be found in the freshwater planarian Schmidtea mediterranae (Sánchez Alvarado, A., and P. A. Tsonis, Nat. Rev. Genet. 2006 7(11):873-84).

The phenomenon of regeneration in biology has captured the imagination of biologists and subsequently the general public since it was described in the 18th century by Ferchault de Reaumur (Mem. Acad. Roy. Sci., pp. 223-245, 1712). For biologists, the ability of animal tissue to regenerate, both structurally and functionally, opened up potentially fruitful lines of research in molecular signal transduction, pattern formation, and plasticity. Research into the mechanisms of biological regeneration is providing crucial insights into many of the basic mechanisms of animal

\footnotetext{
* To whom correspondence should be addressed: E-mail: jcsmith@mbl.edu; jolds@gmu.edu
}

biology, including development, adaptation, immunity, oncogenesis, and even brain mnemonic function.

For the public, regeneration, as a biological phenomenon, offered an existence proof for a type of "healing" that hitherto had been in the realm of religion. As a result, there has been sustained public support for biomedical research on regeneration, overlapping in time the great revolutions in molecular biology of the 20th century. Hence, the field of regeneration research benefited enormously from a synergy in new knowledge, new methods, and new tools from informatics, evolving from its original descriptive roots into a field rich in experimental tradition.

This virtual symposium issue of The Biological Bulletin celebrates a major milestone for our publisher, The Marine Biological Laboratory, as it opens the new Eugene Bell Center for Regenerative Biology and Tissue Engineering on its Woods Hole campus. As with recent virtual symposia published by the journal, the current issue brings together a set of invited reviews, original research reports, and a position paper that offers a coherent and current window into some of the major contemporary trends in animal regeneration research. In addition, and as befitting an issue marking a milestone, we are pleased to republish a landmark research paper on regeneration by T. H. Morgan himself. Originally published in The Biological Bulletin in 1909 (Vol. 18:265276) and entitled "The Dynamic Factor in Regeneration," this work concerns the concept of polarity-specifically, how animals with missing body parts maintain polarity of lost structures during regeneration. Morgan concludes that dynamic processes must constantly function to underpin organismal homeostasis and thus polarity. The mechanisms of polarity maintenance are still not well known. We believe that much in Morgan's paper will be found insightful by 
modern biologists, "equally by students of embryology and by students of regeneration," as Morgan himself might have it.

In organizing the current virtual symposium we have emphasized models and mechanisms. In doing so, however, we caution against oversimplifying. As eukaryotes, animals have a vast diversity of cell types that interact with one another during regeneration under the control of molecular signal-transduction pathways.

In the case of restorative regeneration, the mechanisms of these interactions are typically explored by experiments conducted along the following basic design: An appropriate animal model is chosen. Tissue is damaged or ablated in a controlled fashion. The phenomenon of regeneration is observed under either a control condition (no perturbation) or an experimental condition (where the normal conditions of regeneration have been altered in some systematic way). The experimenter observes the course of the regeneration and correlates changes in that course with the manipulation.

In the case of physiological regeneration, a variation of the above approach is to experimentally perturb the baseline regeneration process during the relevant periods of the animal's life cycle.

In both cases, modern molecular and cellular imaging techniques are often used to observe complex cellular dynamics as they play out. The addition of exploratory approaches (made possible by informatics, genomics, and proteomics) has facilitated progress toward the elucidation of molecular networks and pathways integral to regeneration. The experimental paradigm has expanded in recent years to include comparative phylogenetic and molecular "rescue" approaches.

At the core of this work is the central question of regeneration: why do some animals regenerate while others fail to do so? There is no simple answer to this question. A survey of the literature reveals that even closely related species may differ vastly in their regenerative capabilities. Even so, the current virtual symposium offers some intriguing clues.

Smith et al. in their position paper (pp. 18-34) advocate for the analysis of gene regulatory networks using the lamprey spinal cord system as the model. The advantage of this system, they argue, is that the genome has been sequenced, the means of gene delivery are well established, and the relevance to the problem of central nervous system (CNS) regeneration in other vertebrates is strong because of evolutionary conservation. Smith and colleagues deploy a variety of hypothesis-based and exploratory approaches toward a model of restorative regeneration. The power of the approach lies in the ability to reveal gene regulatory networks that may be conserved in human CNS and may therefore serve as therapeutic targets for new pharmacological and biological compounds after brain or spinal cord injury.

Matsuo and Ito in their paper (pp. 35-42) review regen- eration in neural tissues ranging from mollusc to mammal with particular emphasis on the snail Limax, which has the ability to regenerate its procerebrum without an external intervention. They review both restorative and physiological models for regeneration and implicitly raise the question of whether neuroplasticity, as seen in regeneration, may share molecular mechanisms with learning and memory. They consider the interesting question of how regeneration of the procerebrum, under some circumstances, can restore an engram, when the locus of that engram is normally stored in the same neuroanatomical structure.

Continuing within the context of neuroscience, Turner et al., in an experimental paper (pp. 110-125), present new in vivo and in vitro results on the restorative regeneration and regulation of synaptic transmission at the neuromuscular junction (NMJ) in a snail, Heliosoma. The authors use an electrophysiological approach and hypothesize a homeostatic mechanism for motor system regeneration that includes transient synaptogenesis during axonal outgrowth, inhibitory interneuron synapse formation during formation of restored NMJ, and target-dependent regulation of NMJ formation.

Some of the biggest issues in translational regenerative medicine-the role of stem cells and allorecognitioncome to the fore in studies of regenerative biology in colonial ascidians. Kürn et al. in their paper (pp. 43-61) review the cellular mechanisms controlling the overlapping processes of regeneration and asexual propagation in botryllid ascidians. Colonies of ascidians grow by the asexual propagation of an individual, a process perhaps understandable as an extreme form of physiological regeneration. Indeed, colonial ascidians also possess a capacity for restorative regeneration rivaling the classical example of the planarian. The authors catalog the different cellular players in restorative regeneration and asexual propagation with a focus on Botryllus scholsseri, a particularly tractable system for studying molecular regeneration. In doing so, they highlight the feedback mechanisms that must be present to regulate stem cell activity and detection of self versus non-self.

Though regeneration of the adult nervous system is rare in vertebrates, some of our closest invertebrate cousins within chordates, the ascidians, achieve this feat with apparent ease. Sköld et al. in their research paper (pp. 126136) test the proliferative and morphogenetic properties of cells involved in regeneration in a colonial didemnid ascidian, Diplosoma listerianum, including the cells that have the potential to give rise to an entirely new brain. They also present the first description of the developmental staging of the tissue where the new brain forms, the thoracic budinformation that will help support further investigations in this model system.

The repair and regrowth of fish fins in response to injury has recently emerged as a model of vertebrate regeneration 
that can take advantage of the same characteristics of fish model systems that have proved so useful for developmental investigations. Yoshinari and Kawakami (pp. 62-78) review the process of fin regeneration in zebrafish and medaka, and also discuss studies of regeneration in a number of other vertebrates. This review demonstrates how molecular studies are informing our understanding of the regenerative process by highlighting the "compartments" or subpopulations of cells that are involved in fin regeneration and defined by differential gene expression.

In order to regenerate, the animal (or more properly the cells that possess the potential to repopulate and reform tissue) must detect the injury. It is not difficult in the post-genomics era to consider feedback by signaling systems that then induce differential gene transcription and hence trigger a response. Messerli and Graham in their review (pp. 79-92) discuss a separate mechanism based on endogenously produced DC electric fields. This phenomenon has long been known and researched, with many studies in frogs. Though trials showing that applying DC fields aids in the healing process reach back decades, the cellular and molecular mechanisms by which cells sense and respond to these cues are not known. As application of electric fields has proven clinical effectiveness, one suspects that any mechanistic insights will greatly enhance not only our understanding of the basic biology of the family of injury responses but also our ability to manipulate treatments for producing improved clinical outcomes.

Continuing with frogs, Fukui and Henry in their research paper (pp. 137-145) consider the role of fibroblast growth factor (FGF) signaling in regeneration of the lens in Xenopus. They comprehensively describe the expression patterns of the (numerous) FGF ligands and receptors that, over the course of lens regeneration, are consistent with a function in regulating regenerative processes in this system. They show that addition of an inhibitor of FGF signaling blocks lens regeneration, indicating an essential role for these signals.

One of the most stunning forms of animal regeneration must be that found in sea cucumbers, which under threat of predation eject all of their viscera and subsequently regrow them. Mashanov and García-Arrarás in their review (pp. 93-109) provide an overview of the cellular processes of gut regeneration after evisceration. An age-old question is whether, and to what degree, regenerative processes recapitulate the developmental program. Leaving aside the possibility that different species will give different answers, gut regeneration in sea cucumbers points to a connection between development and regeneration. Sea cucumbers, like most echinoderms, go through a larval stage followed by a radical rearrangement of the body plan during metamorphosis. In sea cucumbers, metamorphosis involves closure of the larval anus and resorption of other intestinal tissues. In one species, however, this degree of remodeling does not take place, and this same species is also incapable of gut regeneration, at any stage. Other features likewise suggest a connection between gut regeneration and developmental processes, though a definitive answer is likely to come only with detailed functional studies. As visceral autotomy followed by regeneration is highly reproducible, this system lends itself well to molecular studies beyond candidate gene approaches. As Mashanov and García-Arrarás point out, this system promises to reach many of the core questions of regenerative biology.

In co-editing the current issue, it became readily apparent to us that the field of regeneration is broad and vibrant. At the same time, the papers here reinforce the notion that the use of animal models to understand the mechanisms of regeneration is both fruitful and of potentially enormous significance to the future practice of medicine. Finally, we were struck anew by how many unanswered questions of basic science are central to regeneration, not the least of which we have already raised above: why do some animals show regeneration while others do not. We hope the present virtual symposium issue of The Biological Bulletin may furnish some clues. 